FACTA UNIVERSITATIS (NIŠ)

Ser. Math. Inform. Vol. 35, No 4 (2020), 1219-1229

https://doi.org/10.22190/FUMI2004219G

\title{
THE LEVINSON-TYPE FORMULA FOR A CLASS OF STURM-LIOUVILLE EQUATION
}

\author{
Sertac Goktas and Khanlar R. Mamedov
}

(C) 2020 by University of Niš, Serbia | Creative Commons Licence: CC BY-NC-ND

Abstract. The boundary value problem

$$
\begin{gathered}
-\psi^{\prime \prime}+q(x) \psi=\lambda^{2} \psi, \quad 0<x<\infty, \\
\psi^{\prime}(0)-\left(\alpha_{0}+\alpha_{1} \lambda\right) \psi(0)=0
\end{gathered}
$$

is considered, where $\lambda$ is a spectral parameter, $q(x)$ is real-valued function such that

$$
\int_{0}^{\infty}(1+x)|q(x)| d x<\infty
$$

with $\alpha_{0}, \alpha_{1} \geq 0\left(\alpha_{0}, \alpha_{1} \in \mathbb{R}\right)$.

In this paper, for above-mentioned boundary value problem, the scattering data is considered and the characteristics properties (such as continuity of the scattering function $S(\lambda)$ and giving the Levinson-type formula) of this data are studied.

Keywords: Scattering data; scattering function; Gelfand-Levitan-Marchenko equation; Levinson-type formula.

\section{Introduction}

Consider the boundary value problem

$$
\begin{gathered}
-\psi^{\prime \prime}+q(x) \psi=\lambda^{2} \psi, \quad 0<x<\infty, \\
\psi^{\prime}(0)-\left(\alpha_{0}+\alpha_{1} \lambda\right) \psi(0)=0,
\end{gathered}
$$

Received November 04, 2019; accepted December 15, 2019

2020 Mathematics Subject Classification. Primary 34B07; Secondary 34A55, 34B24, 34B40, 34L25 
where $q(x)$ is real valued function such that

$$
\int_{0}^{\infty}(1+x)|q(x)| d x<\infty
$$

and $\alpha_{0}, \alpha_{1}$ are real numbers, also $\alpha_{0}, \alpha_{1} \geq 0$.

Spectral analysis when the spectral parameter appearing linearly on the half line for the boundary value problem (1.1) was studied in $[3,4],(1.2)$. In the case $q(x) \equiv$ 0 , this boundary value problem is given by application to the heat transmission problem in [2]. In the wave theory of mathematical physics and geophysics, the applications of the problems can also be found [1, 5, 20,21, 22, 23]. from

$$
e(x, \lambda)=e^{i \lambda x}+\int_{0}^{\infty} K(x, t) e^{i \lambda t} d t,
$$

is a Jost solution of the equation (1.1) for any $\lambda$ on closed upper half plane, where the kernel $K(x, t)$ satisfies the relation

$$
|K(x, t)| \leq \frac{1}{2} \sigma\left(\frac{x+t}{2}\right) \exp \left\{\sigma_{1}(x)-\sigma_{1}\left(\frac{x+t}{2}\right)\right\}
$$

with

$$
\sigma(x) \equiv \int_{x}^{\infty}|q(t)| d t, \quad \sigma_{1}(x) \equiv \int_{x}^{\infty} \sigma(t) d t
$$

and

$$
K(x, x)=\frac{1}{2} \int_{x}^{\infty} q(t) d t .
$$

The function $e(x,-\lambda)$ satisfies the equation (1.1) for each $\lambda \in \mathbb{R} \backslash\{0\}$ and the functions $e(x, \lambda)$ and $e(x,-\lambda)$ form a fundamental set of solutions for the differential equation (1.1). Their Wronskian is as follows:

$$
W\{e(x, \lambda), e(x,-\lambda)\}=e^{\prime}(x, \lambda) e(x,-\lambda)-e(x, \lambda) e^{\prime}(x,-\lambda)=2 i \lambda .
$$

Let $\varpi(x, \lambda)$ denote the a special solution of the equation (1.1) that satisfies the initial conditions

$$
\varpi(0, \lambda)=1, \quad \varpi^{\prime}(0, \lambda)=\alpha_{0}+\alpha_{1} \lambda .
$$

The following lemma 1.1 and lemma 1.2 which have been proved in [9] should be given in order to achieve the aim of the manuscript: 
Lemma 1.1. The identity

$$
\frac{2 i \lambda \varpi(x, \lambda)}{e^{\prime}(0, \lambda)-\left(\alpha_{0}+\alpha_{1} \lambda\right) e(0, \lambda)}=e(x,-\lambda)-S(\lambda) e(x, \lambda)
$$

holds for all real $\lambda \neq 0$ where

$$
S(\lambda)=\frac{e^{\prime}(0,-\lambda)-\left(\alpha_{0}+\alpha_{1} \lambda\right) e(0,-\lambda)}{e^{\prime}(0, \lambda)-\left(\alpha_{0}+\alpha_{1} \lambda\right) e(0, \lambda)}
$$

and

$$
|S(\lambda)|=1 .
$$

Here, the function $S(\lambda)$ is represented by the formula (1.5). This function is called the scattering function of the boundary value problem (1.1)-(1.3).

The function $S(\lambda)$ is meromorphic function on the upper half plane $(\operatorname{Im} \lambda>0)$. The zeros of the function $\varphi(\lambda) \equiv e^{\prime}(0, \lambda)-\left(\alpha_{0}+\alpha_{1} \lambda\right) e(0, \lambda)$ are the poles of the function $S(\lambda)$.

Lemma 1.2. The function $\varphi(\lambda)$ may have only a finite number of zeros $\lambda_{1}, \lambda_{2}, \ldots, \lambda_{n}$ on the half plane Im $\lambda>0$ and all these zeros don't lie on the imaginary axis. The zeros $\varphi(\lambda)$ and $\varphi_{1}(\lambda) \equiv e^{\prime}(0,-\lambda)-\left(\alpha_{0}+\alpha_{1} \lambda\right) e(0,-\lambda)$ are complex conjugate each other and the number of these zeros is equal.

The number $m_{k}$ is referred to the multiplicity of the zeros $\lambda_{k},(k=1,2, \ldots, n)$ of the equation $\varphi(\lambda)=0$. These $\lambda_{k}$ is called the singular values of the boundary value problem (1.1)-(1.3).

We denote

$$
f_{j}(x)=i \operatorname{Res}_{\lambda=\lambda_{j}} \frac{\varphi_{2}(\lambda)}{\varphi(\lambda)} e^{i \lambda x},
$$

where $\varphi_{2}(\lambda)=\hat{e}^{\prime}(0, \lambda)-\left(\alpha_{0}+\alpha_{1} \lambda\right) \hat{e}(0, \lambda)$ and $\hat{e}(x, \lambda)$ is a solution of the equation (1.1) (see $[18$, p.299]). We shall call the polynomial

$$
P_{k}(x)=e^{-i \lambda_{k} x} f_{k}(x), \quad k=1,2, \ldots, n,
$$

with degree of $m_{k}-1$ the normalization poliynomial for boundary value problem (1.1)-(1.3).

Let

$$
\begin{gathered}
F_{s}(x)=\frac{1}{2 \pi} \int_{-\infty}^{\infty}\left[S_{0}-S(\lambda)\right] d \lambda, \\
F(x)=\sum_{k=1}^{n} f_{k}(x)+F_{s}(x),
\end{gathered}
$$

where $S_{0}=\frac{a+i}{a-i}$. 
The kernel function $K(x, t)$ of the special solution (1.4) satisfies the integral equation

$$
F(x+y)+K(x, y)+\int_{x}^{\infty} K(x, t) F(t+y) d t=0, \quad x<y<\infty
$$

for each $x \geq 0$.

The equation (1.7) is called the main equation (also called Gelfand-LevitanMarchenko equation) of the inverse boundary value problem (1.1)-(1.3). This main equation admits a uniquely solution $K(x, t)$ in the space $L_{1}(x, \infty)[9]$.

The set of values $\left\{S(\lambda), \lambda_{k}, P_{k}(x),(k=\overline{1, n})\right\}$ is referred to as the scattering data of the boundary value problem (1.1)-(1.3) (see [8]). The inverse scattering problem consists in uniquely recovering the coefficient $q(x)$ from the scattering data. Given the scattering data, we can use formula (1.6) to construct the function $F(x)$ and write out to main equation (1.7) for the unknown function $K(x, y)$. The main equation has a unique solution for every $x \geq 0$. Solving this equation, we find the kernel $K(x, y)$ of the solution (1.7) and hence potential $q(x)=-2 \frac{d K(x, x)}{d x}$.

Note that the inverse problem of scattering theory on the half line for the boundary value problem (1.1)-(1.3) in the case $\alpha_{1}=0$ was completely solved in $[6,7,15,16]$. Inverse problems in the half line with spectral parameter contained in the boundary conditions was investigated according to spectral function in [19], according to Weyl function in [21]-[23], and acording to scattering data [10]-[13]. In the case of non-selfadjoint, the similar problem was solved in [8]. The uniqueness of solution of inverse scattering problem for boundary value problem (1.1)-(1.3) is given in [9] by using the methods of [8] and [15]. Different from the classical case the zeros of Jost function not lie imaginary axis, lie complex plane and these zeros not simple. The boundary value problem (1.1)-(1.3) is not selfadjoint and for this reason, scattering data is differently defined. Therefore, the properties of the scattering data have to be investigated. The present work is devoted to give the properties of the scattering data of boundary problem (1.1)(1.3). Similar problem in the self-adjoint case was studied in [14, 17].

Let us give a brief description of the structure of our study. In Section 2, we prove the continuity of the scattering function on the whole axis. In Section 3, we derive the Levinson type formula.

\section{The continuity of the scattering function}

In this section, the continuity of the scattering function $S(\lambda)$ defined by (1.5) will be investigated.

Theorem 2.1. The scattering function $S(\lambda)$ is continuous for all real points $\lambda$.

Proof. It follows from lemma 1.1 that $\varphi(\lambda) \neq 0$ for all $\lambda \neq 0$. The continuity of the function $S(\lambda)$ can be obtained from hence. 
When $\varphi(0) \neq 0$, the function $S(\lambda)$ is continuous for $\lambda=0$ and $S(0)=1$.

Let $\varphi(0)=0$. Namely,

$$
\begin{aligned}
\varphi(0) & =e^{\prime}(0,0)-\alpha_{0} e(0,0) \\
& =-K(0,0)+\int_{0}^{\infty} K_{x}(0, t) d t-\alpha_{0}\left[1+\int_{0}^{\infty} K(0, t) d t\right]=0 .
\end{aligned}
$$

To complete proof, we shall investigate the continuity of the function $S(\lambda)$ in this case.

Now, putting $x=0$ in the main equation (1.7), we obtain

$$
K(0, y)+F(y)+\int_{0}^{\infty} K(0, t) F(t+y) d t=0
$$

Substituting $x=0$ after differentiating the main equation (1.7) with respect to $x$, we get

$$
K_{x}(0, y)+F^{\prime}(y)-K(0,0) F(y)+\int_{0}^{\infty} K_{x}(0, t) F(t+y) d t=0
$$

After multiplying the equation (2.2) throughout by $-\alpha_{0}$ and adding to the equality (2.3), we have

$$
K_{x}(0, y)-\alpha_{0} K(0, y)-\left(\alpha_{0}+K(0,0)\right) F(y)+F^{\prime}(y)+\int_{0}^{\infty}\left[K_{x}(0, t)-\alpha_{0} K(0, t)\right] F(t+y) d t=0 .
$$

Then, integrating the equality (2.4) with respect to $y$ from $z$ to $\infty$, we obtain

$$
\begin{aligned}
\int_{z}^{\infty}\left[K_{x}(0, y)-\alpha_{0} K(0, y)\right] d y & -\left(\alpha_{0}+K(0,0)\right) \int_{z}^{\infty} F(y) d y-F(z) \\
& +\int_{0}^{\infty}\left[K_{x}(0, t)-\alpha_{0} K(0, t)\right] \int_{z+t}^{\infty} F(\xi) d \xi d t=0 .
\end{aligned}
$$

Put $K_{1}(z)=\int_{z}^{\infty}\left[K_{x}(0, y)-\alpha_{0} K(0, y)\right] d y$. Then, from the last equality, the following relation is obtained:

$$
K_{1}(z)-\left(\alpha_{0}+K(0,0)\right) \int_{z}^{\infty} F(y) d y-F(z)-\int_{0}^{\infty}\left(\int_{z+t}^{\infty} F(\xi) d \xi\right) d K_{1}(t)=0 .
$$


Using integration by parts and considering the following process

$$
\begin{aligned}
&\left.\int_{0}^{\infty} K_{x}^{\prime}(x, t)\right|_{x=0} \int_{t+z}^{\infty} F(\xi) d \xi d t=\left.\int_{0}^{\infty} K_{x}^{\prime}(x, t)\right|_{x=0} \int_{z}^{\infty} F(y) d y d t \\
&-\left.\int_{0}^{\infty} F(t+z) \int_{t}^{\infty} K_{x}^{\prime}(x, \xi)\right|_{x=0} d \xi d t \\
& \int_{z}^{\infty} F^{\prime}(y) d y-K(0,0) \int_{z}^{\infty} F(y) d y+\left.\int_{z}^{\infty} K_{x}^{\prime}(x, y)\right|_{x=0} d y \\
&+\left.\int_{0}^{\infty} K_{x}^{\prime}(x, t)\right|_{x=0} \int_{z}^{\infty} F(y) d y d t-\left.\int_{0}^{\infty} F(t+z) \int_{t}^{\infty} K_{x}^{\prime}(x, \xi)\right|_{x=0} d \xi d t=0,
\end{aligned}
$$

we get

$$
K_{1}(z)-\left(\alpha_{0}+K(0,0)+K_{1}(0)\right) \int_{z}^{\infty} F(y) d y-F(z)-\int_{0}^{\infty} K_{1}(t) F(t+z) d t=0 .
$$

Hence, when $\varphi(0)=0$ (from $(2.1)), K_{1}(z)$ is the bounded solution of the equation

$$
K_{1}(z)-\int_{0}^{\infty} K_{1}(t) F(t+z) d t=F(z), \quad(0 \leq z<\infty) .
$$

It is evident that the bounded solution of this equation is integrable on the half axis. It means that $K_{1}(z) \in L_{1}(0, \infty)$ (see [15], p. 211).

Returning to the representation $\varphi(\lambda)$, we have

$$
\begin{aligned}
\varphi(\lambda) & =i \lambda-K(0,0)+\int_{0}^{\infty} K_{x}(0, t) e^{i \lambda t} d t-\left(\alpha_{0}+\alpha_{1} \lambda\right)\left[1+\int_{0}^{\infty} K(0, t) e^{i \lambda t} d t\right] \\
& =i \lambda K(0,0)+\int_{0}^{\infty} K_{x}(0, t) e^{i \lambda t} d t-\alpha_{0}\left[1+\int_{0}^{\infty} K(0, t) e^{i \lambda t} d t\right] \\
& -\alpha_{1} \lambda\left[1+\int_{0}^{\infty} K(0, t) e^{i \lambda t} d t\right]
\end{aligned}
$$

where 


$$
\begin{aligned}
& -K(0,0)+\int_{0}^{\infty} K_{x}(0, t) e^{i \lambda t} d t-\alpha_{0}-\alpha_{0} \int_{0}^{\infty} K(0, t) e^{i \lambda t} d t= \\
& =-K(0,0)-\int_{0}^{\infty} e^{i \lambda t} d\left(\int_{t}^{\infty} K(0, y) d y\right)-\alpha_{0}+\alpha_{0} \int_{0}^{\infty} e^{i \lambda t} d\left(\int_{t}^{\infty} K(0, y) d y\right) \\
& =-K(0,0)+\int_{x}^{\infty} K_{x}(0, y) d y-\alpha_{0} \int_{0}^{\infty} K(0, y) d y+i \lambda \int_{0}^{\infty} e^{i \lambda t} \int_{t}^{\infty} K_{x}(0, y) d y d t \\
& -i \alpha_{0} \lambda \int_{0}^{\infty} e^{i \lambda t} \int_{0}^{\infty} K(0, y) d y d t \\
& =i \lambda \int_{0}^{\infty} \int_{t}^{\infty}\left(K_{x}(0, y)-\alpha_{0} K(0, y)\right) d y e^{i \lambda t} d t \\
& =i \lambda \int_{0}^{\infty} K_{1}(t) e^{i \lambda t} d t .
\end{aligned}
$$

Hence, we obtain

$$
\begin{aligned}
\varphi(\lambda) & =i \lambda\left[1+\int_{0}^{\infty} K_{1}(t) e^{i \lambda t} d t-i \alpha_{1}\left(1+\int_{0}^{\infty} K(0, t) e^{i \lambda t} d t\right)\right] \\
& =i \lambda \widetilde{K}(\lambda) .
\end{aligned}
$$

where

$$
\widetilde{K}(\lambda)=1-i \alpha_{1}+\int_{0}^{\infty} K_{1}(t) e^{i \lambda t} d t-i \alpha_{1} \int_{0}^{\infty} K(0, t) e^{i \lambda t} d t .
$$

Similarly, we get

$$
\varphi_{1}(\lambda)=-i \lambda \widetilde{K_{1}}(\lambda)
$$

where

$$
\widetilde{K_{1}}(\lambda)=1+i \alpha_{1}+\int_{0}^{\infty} K_{1}(t) e^{-i \lambda t} d t-i \alpha_{1} \int_{0}^{\infty} K(0, t) e^{-i \lambda t} d t .
$$

Consequently, from the equality (1.5)

$$
S(\lambda)=-\frac{\widetilde{K_{1}}(\lambda)}{\widetilde{K}(\lambda)}
$$


Taking into account lemma 1.1 (see [9]) and by using the formulas (2.5) and (2.6), we can write

$$
2 \varpi(x, \lambda)=\widetilde{K}(\lambda)[e(x,-\lambda)-S(\lambda) e(x, \lambda)] .
$$

It can be seen that $\widetilde{K}(\lambda) \neq 0$, otherwise it would be $\varphi(x, 0)=0$. But, this can not be true since $\varphi(0,0)=1$. So, $S(\lambda)$ is continuous at $\lambda=0$ and by condition (2.1) it holds $S(\lambda)=-\frac{\widetilde{K_{1}}(0)}{\widetilde{K}(0)}$.

This completes the proof the theorem.

\section{The Levinson-Type formula}

We give the formula that expresses the relation between the increment of the argument of the scattering function $S(\lambda)$ and the singular number $\lambda_{k}$ of boundary value problem (1.1)-(1.3).

Theorem 3.1. The following formlua is valid:

$$
-\frac{1-S(0)}{2}-\left.\frac{1}{2 \pi} \arg S(\lambda)\right|_{-\infty} ^{\infty}+1=2\left[m_{1}+m_{2}+\ldots+m_{n}\right],
$$

where $m_{k}(k=1,2, \ldots, n)$ is the multiplicity of the singular number $\lambda_{j}(j=1,2, \ldots, n)$.

Proof. For sufficiently little $\varepsilon>0$ and sufficiently large $R>0$, let

$$
\Gamma_{R, \varepsilon}=C_{R}^{+} \cup C_{\varepsilon}^{-} \cup[-R,-\varepsilon] \cup[\varepsilon, R],
$$

where $C_{R}^{+}$and $C_{\varepsilon}^{-}$are circles with centers in origin and corresponding radius of $R$ and $\varepsilon$, respectively (Fig. 1). Orientation on the $C_{R}^{+}$is positive and on the $C_{\varepsilon}^{-}$ negative.

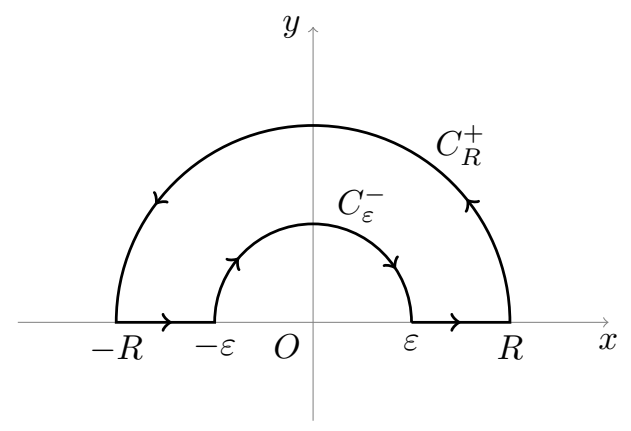

Figure 1: The Graph of $\Gamma_{R, \varepsilon}$. 
Let us apply argument principle to $\varphi(\lambda)$ function. This function is regular on the upper half plane and continuous on the closed half plane $\operatorname{Im} \lambda \geq 0$. When moving from $-\infty$ to $\infty$ on the whole real axis and passing origin from top along with half circle with radius $\varepsilon$, the change in the argument of $\varphi(\lambda)$ is equal to number of its pole points times $2 \pi$ :

$$
\left.\arg \varphi(\lambda)\right|_{[-R,-\varepsilon] \cup[\varepsilon, R]}+\left.\arg \varphi(\lambda)\right|_{\Gamma_{\varepsilon}}+\left.\arg \varphi(\lambda)\right|_{\Gamma_{R}}=2 \pi\left[m_{1}+m_{2}+\ldots+m_{n}\right]
$$

or

$$
\frac{1}{2 \pi i}\left(\int_{C_{R}^{+}}+\int_{C_{\varepsilon}^{-}}+\int_{\varepsilon}^{R}+\int_{-R}^{-\varepsilon}\right) d \ln \varphi(\lambda)=m_{1}+m_{2}+\ldots+m_{n} .
$$

From (1.5), the scattering function $S(\lambda)$ has the form

$$
S(\lambda)=\frac{\varphi_{1}(\lambda)}{\varphi(\lambda)}
$$

for real $\lambda$. It is clear from here that $\arg S(\lambda)=-2 \arg \varphi(\lambda)$.

Using the last equality, we have

$$
\left.\arg \varphi(\lambda)\right|_{[-R,-\varepsilon] \cup[\varepsilon, R]}=-\frac{1}{2} \arg S(\lambda) .
$$

Considering (3.3) in the equality (3.2), we obtain

$$
-\left.\frac{1}{2} \arg S(\lambda)\right|_{[-R,-\varepsilon] \cup[\varepsilon, R]}+\left.\arg \varphi(\lambda)\right|_{C_{\varepsilon}^{-}}+\left.\arg \varphi(\lambda)\right|_{C_{R}^{+}}=2 \pi\left[m_{1}+m_{2}+\ldots+m_{n}\right]
$$

According to theorem 2.1, the function $S(\lambda)$ is continuous on the whole real axis. Hence,

$$
\begin{aligned}
& \lim _{\substack{R \rightarrow \infty \\
\varepsilon \rightarrow 0}}\left\{-\left.\frac{1}{2} \arg S(\lambda)\right|_{[-R,-\varepsilon] \cup[\varepsilon, R]}\right\}=-\left.\frac{1}{2} \arg S(\lambda)\right|_{-\infty} ^{\infty}, \\
& \left.\lim _{\varepsilon \rightarrow 0} \arg \varphi(\lambda)\right|_{C_{\varepsilon}^{-}}=\left\{\begin{array}{cc}
0, & \text { if } \varphi(0) \neq 0, \\
-\pi, & \text { if } \varphi(0)=0,
\end{array}=-\frac{\pi(1-S(0))}{2}\right.
\end{aligned}
$$

and

$$
\lim _{R \rightarrow \infty} \arg \varphi(\lambda)=\pi
$$


from lemma 1.1 . have

Taking into account the equalities (3.5), (3.6) and (3.7) in the equality (3.4), we

$$
-\left.\frac{1}{2} \arg S(\lambda)\right|_{-\infty} ^{\infty}+\pi+\left\{\begin{array}{rl}
0, & \text { if } \varphi(0) \neq 0, \\
-\pi, & \text { if } \varphi(0)=0,
\end{array}=2 \pi\left[m_{1}+m_{2}+\ldots+m_{n}\right]\right.
$$

From this last equality, the formula (3.1) is obtained, which proves the theorem.

The note that, this formula is called the Levinson-type formula for the boundary value problem (1.1)-(1.3).

\section{REF EREN CES}

1. S. A. Alimov: A. N. Tihonov's works on inverse problems for the Sturm-Liouville equation. Matematicheskikh Nauk, 31 (6) (1976) 84-88; English Trans: English translation in Russian Mathematical Surveys Uspekhi 31 (6) (1976) 87-92;

2. D. S. CoHen: An integral transform associated with boundary conditions containing an eigenvalue parameter. SIAM Journal on Applied Mathematics, 14 (5) (1966) 11641175.

3. C. T. Fulton: Singular eigenvalue problems with eigenvalue parameter contained in the boundary conditions. Proceedings of the Royal Society of Edinburgh. Section A, 87 (1-2) (1980) 1-34.

4. C. T. Fulton: Two point boundary value problems with eigenvalue parameter contained in the boundary conditions. Proceedings of the Royal Society of Edinburgh Section A. 77 (3-4) (1977) 293-308.

5. G. Freiling, V. A. Yurko: Inverse Sturm-Liouville problems and their applications. NOVA Science Publishers, New York, 2001.

6. B. M. LEVITAN: On the solution of the inverse problem of quantum scattering theory. Mathematical Notes, 17 (4) (1975) 611-624.

7. B. M. Levitan: Inverse Sturm-Liouville Problems. VSP, Zeist:The Netherlands, 1987.

8. V. E. LyANTSE: On a differential equations with spectral singularities I. Matematicheskii Sbornik, 106 (4) (1964) 521-561.

9. KH. R. MAmedov: On the inverse problem for Sturm-Liouville operator with a nonlinear spectral parameter in the boundary condition. Journal of Korean Mathematical Society, 46 (6) (2009) 1243-1254.

10. KH. R. MAmedov: Uniqueness of the solution of the inverse problem of scattering theory for the Sturm-Liouville operator with a spectral parameter in the boundary condition. Mathematical Notes, 74 (1-2) (2003) 136-140. 
11. KH. R. Mamedov: On an inverse scattering problem for a discontinuous SturmLiouville equation with a spectral parameter in the boundary condition. Boundary Value Problems, 2010 (2010), doi:10.1155/2010/171967.

12. KH. R. MAMEdov AND A. ÇöL: On the inverse problem of scattering theory for a class of systems of Dirac equations with discontinuous coefficient. European Journal of Pure and Applied Mathematics, 1 (3) (2008) 21-32.

13. KH. R. MAmedov, A. ÇÖL: On an inverse scattering problem for a class Dirac operator with discontinuous coefficient and nonlinear dependence on the spectral parameter in the boundary condition. Mathematical Methods in the Applied Sciences, 35 (14) (2012) 1712-1720.

14. Kн. R. Mamedov, H. Menken: The Levinson-type formula for a boundary value problem with a spectral parameter in the boundary condition. The Arabian Journal of Science and Engineering, 34 (1A) (2009) 219-226.

15. V. A. MARChenko: Sturm-Liouville operators and applications, vol.22 of Operator theory: Advances and applications. Birkhauser: Basel, Switzerland, 1986.

16. V. A. Marchenko: On reconstruction of the potential energy from phases of the scattered waves. Doklady Akademii Nauk SSSR, 104 (1955) 695-698.

17. Ö. Mizrak, Kh. R. Mamedov, A. M. Akhtyamov: Characteristic properties of scattering data of a boundary value problem. Filomat, 31 (12) (2017) 3945-3951.

18. M. A. NAImARK: Linear Differential Operators. Vol. II: Linear Differential Operators in Hilbert Space. George G. Harrap \& Company Limited: London, 1968.

19. E. A. PocheykinA-Fedotova: $n$ the inverse problem of boundary problem for second order differential equation on the half line. Izvestiya Vuzov, 17 (1972) 75-84.

20. A. N. Tikhonov, A. A. SAmarskis: Equations of mathematical physics. Pergamon:Oxford, 1963.

21. V. A. YuRKo: Reconstruction of pencils of differential operators on the half-line. Mathematical Notes, 67 (2) (2000) 261-265.

22. V. A. YURKO: An inverse problem for differential operator pencils. Sbornik: Mathematics, 191 (10) (2000) 1561-1586.

23. V. A. YURKO: Method of spectral mappings in the inverse problem theory, inverse and ill-posed problem series. VSP, Utrecht, Netherlands, 2002.

Sertac Goktas

Faculty of Science and Letters

Department of Mathematics

33343, Mersin, Turkey

srtcgoktas@gmail.com

Khanlar R. Mamedov

Faculty of Science and Letters

Department of Mathematics

33343, Mersin, Turkey

hanlar@mersin.edu.tr 